\title{
Hydrodynamic Properties of Water in Myoplasm in Resting and Active States
}

\author{
By Michi-hiko OGATA \\ The Institute of Health Science, Kyushu University 11, 6-1 Kasuga Koen, Kasuga 816 \\ (Communicated by Setsuro EBASHI, M. J. A., June 11, 1996)
}

\begin{abstract}
As indicated by the experiment in early days (Ogata, 1965), the electrical resistance of the myoplasm in resting state is very high compared with that in activated state. This is in accord with the observation that water is only slowly squeezed out from relaxed muscle fragments by applying pressure, compared with that from the activated muscle fragments. All these findings suggest that the water in the relaxed fiber is well retained by structural proteins in accord with the structure of water in resting muscle demonstrated by ${ }^{1} \mathrm{H}-\mathrm{NMR}$ studies.
\end{abstract}

Key words : Viscosity; muscle contraction; water structure of myoplasm; electrical resistance of myoplasm.

Introduction. More than 30 years ago it was found that during sustained contraction of frog sartorius, the myoplasmic electrical resistance fell to less than half of that in the resting state. ${ }^{1), 2}$ A question was then raised what kind of physicochemical change would take place as the background of this phenomenon.

The electrical conductance in the myoplasm is mediated by electrolytes and there is no possibility for the drastic change in the number of electrolytes to explain this marked fall. Since the electrical resistance in the myoplasm is definitely higher than that in the nerve and the electrical conductance does not change drastically during excitation of the nerve, it is plausible to assume that the change in the state of structural proteins of muscle during contraction would play leading part in the above phenomenon.

With this thought in mind, I have been conducting various kinds of experiments to elucidate the mechanism of the change in hydrodynamic state in the myoplasm.

It is a remarkable fact that virtually no water will not leak out from the transverse section of muscle fiber in the resting state and if muscle is activated, water is now easily pushed out.

In this article emphasis will be placed on the kinetic analysis of the water leakage from the transverse section of muscle. Some other experiments that are useful for the explanation of the change in the structure of water in muscle during contraction will also be referred to.

Results. Preliminary experiments revealed that the amount of leakage from 0 to 5 minutes increased proportionally to the compression, and differed markedly when muscle fragments (MF) were activated by fine powder of caffeine (Fig. 1). Temperature coefficient $\left(\mathrm{Q}_{10}\right)$ for the leakage rate was estimated to be 0.8 (from data at $23^{\circ} \mathrm{C}$ and $5^{\circ} \mathrm{C}$ ).

The rate of weight loss caused by squeezing cell water from the MF are plotted against time in Fig. 2. The time constants $(\tau)$ computed by an exponential regression analysis are summarized as follows:

\begin{tabular}{ccc}
\hline Pressure & Relaxed & Activated \\
\hline$\left[\mathrm{kg} / \mathrm{cm}^{2}\right]$ & $\tau$ [sec] & $\tau$ [sec] \\
2 & 1174 & 222 \\
5 & 751 & 145 \\
8 & 534 & 104 \\
\hline
\end{tabular}

By $\mathrm{j}_{\mathrm{t}}$ we denote the amount of leakage at time t. For theoretical consideration it is natural to assume;

$$
\mathrm{j}_{\mathrm{t}}=\mathrm{j}_{\infty} \times\{1-\exp (-\mathrm{t} / \tau)\}
$$

where $j_{\infty}$ [gmwater] means the total loss of water, and 

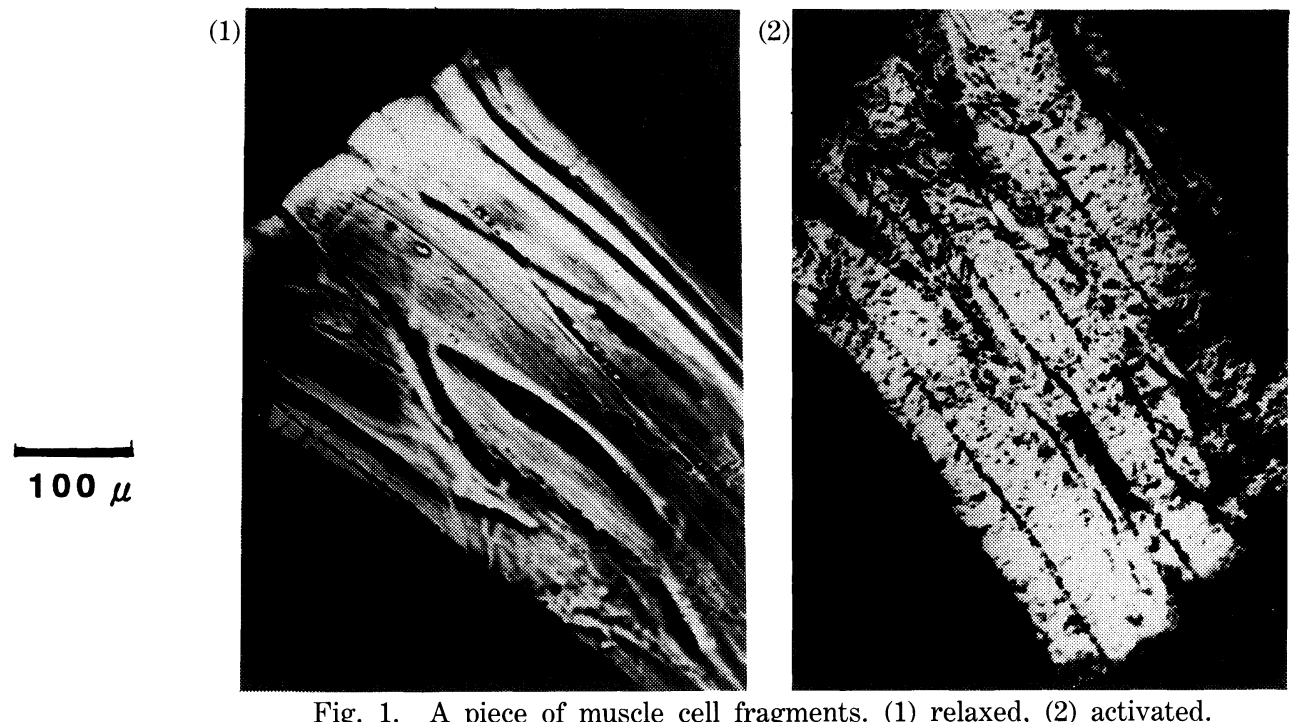

Fig. 1. A piece of muscle cell fragments. (1) relaxed, (2) activated.

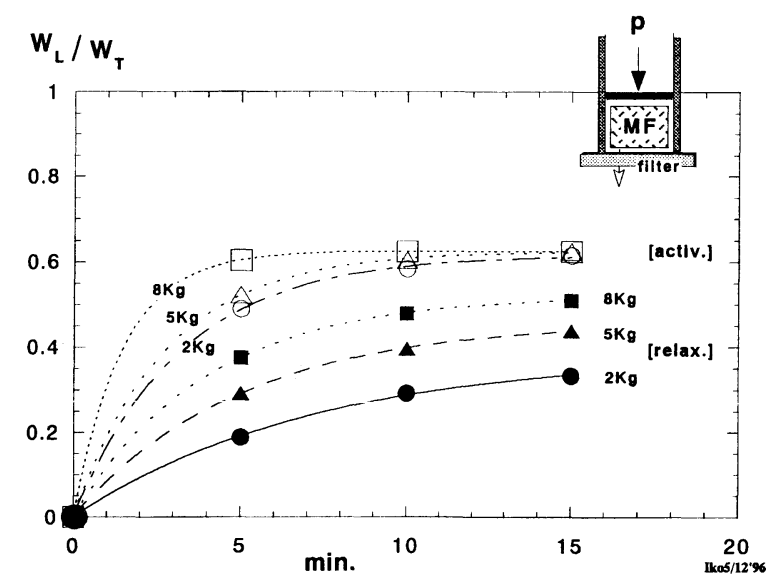

Fig. 2. Relative weight loss of MF against time. Closed symbols = relaxed MF, open symbols=activated MF. Small number shows applied pressure. Above right is a sketch of method.

$\tau$ [sec] is a time constant.

From the experiment it was observed that the average of the final weight ratio, $\left(\mathrm{MF}_{\text {solid }}\right) /\left(\mathrm{MF}_{\text {total }}\right)$, was 0.37 (data not shown). This means the total amounts of leakage are the same both in relaxed and activated. Therefore, it seems reasonable that the total loss of water $\left(\mathrm{W}_{\text {Loss }}\right)$ is regarded as a constant (63\% of initial weight).

$J_{t}$ increases and at $t=7 \times \tau$ reaches almost $j_{\infty}$. The leakage velocity $\left(j^{\prime} t\right.$, [gmwater/sec]) is expressed as follows:

$$
\mathrm{j}_{\mathrm{t}}^{\prime}=\left(\mathrm{j}_{\infty} / \tau\right) \times \exp (-\mathrm{t} / \tau)
$$

Experimental values of $\tau$ under different experimental conditions shown above are put in the equation [2] and $j^{\prime}{ }_{t}$ are computed. Since MF is squeezed, $j_{t}{ }_{t}$ depends on pressure, $p$. So let us denote it by $J(p)$. From each experimental value $J(p)$, we can see $J(p)$ is linear, that is,

$$
J(p)=J(0)+\zeta^{-1} \times p
$$

where $J(0)$ and $\zeta^{-1}$ are constant. Dimensions of $J(p)$, $\zeta^{-1}$ and $\mathrm{p}$ are $\mathrm{gm} / \mathrm{sec}, \mathrm{gm} / \mathrm{kg}$ sec and $\mathrm{kg} / \mathrm{cm}^{2}$. Here are estimations of $\mathrm{J}(0),\left[10^{-4} \mathrm{gm} / \mathrm{sec}\right]$, and $\zeta^{-1} \mathrm{sec},\left[10^{-4}\right.$ $\mathrm{gm} / \mathrm{kg} \mathrm{sec}]$ :

\begin{tabular}{crc}
\hline & $\mathrm{J}(0)$ & $\zeta^{-1}$ \\
\hline Relaxed & 5.01 & 1.70 \\
Activated & 27.46 & 8.52 \\
\hline
\end{tabular}

Although equation [1] assumes a single regression, MF sample still maintains intact subcellular structures. It was then attempted to assume the presence of two regressions.

$$
\mathrm{j}_{\mathrm{t}}=\left(\mathrm{j}_{\infty}, \mathrm{b}\right) \times\left\{\mathrm{b}-\exp \left(-\mathrm{t} / \tau_{1}\right)-(\mathrm{b}-1) \times \exp \left(-\mathrm{t} / \tau_{2}\right)\right\}
$$

where $\mathrm{b}$ is some population factor, and $\tau_{1}$ and $\tau_{2}$ are regression coefficients.

Eq. [4] means that there are two groups of water in the MF. Coefficient $\tau_{2}$ is more closely related to the 


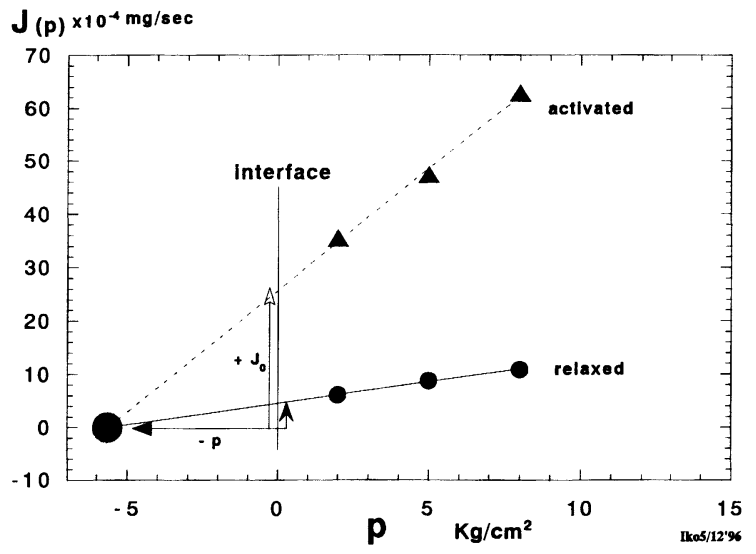

Fig. 3. Relation between leakage velocity and pressure. Note positive water flux $\left(+J_{0}\right)$, negative pressure $(-p)$ at the interface, transverse section of muscle fiber.

contractile system than the other. Results for $\mathrm{p}=2 \mathrm{~kg}$ are as follows:

\begin{tabular}{ccccccc}
\hline & \multicolumn{3}{c}{ Relaxed } & \multicolumn{4}{c}{ Activated } \\
\hline $\mathrm{b}$ & $\tau_{1}$ & $\tau_{2}$ & $\mathrm{~b}$ & $\tau_{1}$ & $\tau_{2}$ & [sec] \\
5 & 176 & 1650 & 2 & 116 & 282 & \\
\hline
\end{tabular}

This table shows that the $\tau_{1}$ water group is less structured than the $\tau_{2}$ water group. Fig. 3 shows how $J(p)$ and $p$ are related in the $\tau_{2}$ water group. In the multiple regressions $J(0)$ and $\zeta^{-1}$ are estimated as follows;

\begin{tabular}{crc}
\hline & $\mathrm{J}(0)$ & $\zeta^{-1}$ \\
\hline Relaxed & 4.54 & 0.80 \\
Activated & 25.72 & 4.57 \\
\hline
\end{tabular}

From experimental values of $\tau, J(p)$ is computed, and then $J(0)$ and $\zeta^{-1}$ are estimated. Results of the cases of single and multiple regressions are essentially the same.

There are three things to be mentioned:

(a) Viscosity, $\zeta$, is reduced in activated state.

(b) $\mathrm{J}(0)$ is positive both in relaxed and activated.

This suggests that the water tends to leak out without any external acceleration (at $\mathrm{p}=0 \mathrm{~kg}$ ). (c) If $J(p)=0$, then $p=-J(0) / \zeta^{-1}<0$. (negative $p$ ).

Theoretical negative pressure is about $-3 \mathrm{~kg}$ (single regression) and $-5.6 \mathrm{~kg} / \mathrm{cm}^{2}$ (multiple regressions). Since the MF is squeezed, outward flux of water, J(p), is positive. Negative pressure would act for counterpoising the positive water flux at the transverse section of muscle, a simple interface.

Myoplasmic electrical resistance in relaxed and activated fibers. This experiment was the very beginning of the series of this research. ${ }^{1,2)}$ In the following the old experiment was confirmed.

Three intracellular electrodes, one for current application and others for voltage recording, are inserted into a single muscle fiber. The diameter of a fiber and distance between electrodes are measured microscopically (100-400x). By applying a weak rectangular pulse, specific values of resistance regarding a muscle fiber as a distributed constant circuit are computed. In the relaxed muscle (m. sartorius of frog), $\mathrm{Rm}$ (membrane) and $\mathrm{Ri}$ (myoplasm) are $2790 \Omega / \mathrm{cm}^{2}$ and $242 \Omega \mathrm{cm}$.

Slowly increasing current applied through an intracellular electrode activates the fiber locally without producing conductive impulse. Mechanical events, changes of striation pattern, shortening and volume, are observed and recorded microscopically. When the activating current is fixed at the supra-threshold level, local shortening stays in a stable condition (sustained activated state, SA). Under this $\mathrm{SA} \operatorname{Re}$ and $\lambda$ are estimated. As a result $\mathrm{Rm}$ and $\mathrm{Ri}$ are calculated to be $2450 \Omega / \mathrm{cm}^{2}$ and $109 \Omega \mathrm{cm}$. Ri decreased considerably ( $45 \%$ of relaxed $\mathrm{Ri}){ }^{1)}$

Direct measurement with skinned fibers (frog) gives $263 \Omega \mathrm{cm}$ in rest, and $156 \Omega \mathrm{cm}$ in activated. ${ }^{2)}$

Measurements were also made with the minced muscle (frog), glycerinated muscle (rabbit), glycerinated fibre bundles (water bug), fresh muscle homogenate (rabbit), myofibril suspension (rabbit), using impedance bridge. Various ways of activation such as the application of caffeine, $\mathrm{Ca}^{2+}$ or ATP were employed.

The impedance of the activated sample $(\mathrm{Za})$ is always lower than in the relaxed state ( $\mathrm{Zr}$ ), if the ionic strength ranged from 0.15 to 0.3 . Data are summarized in Fig. 4.

Conductance of aqueous solution with similar ionic strength is about four times higher than Ri.

Impedance of contractile protein suspensions. The impedance of actin, myosin and actomyosin suspensions, $(\mathrm{Zs})$, are measured under different 


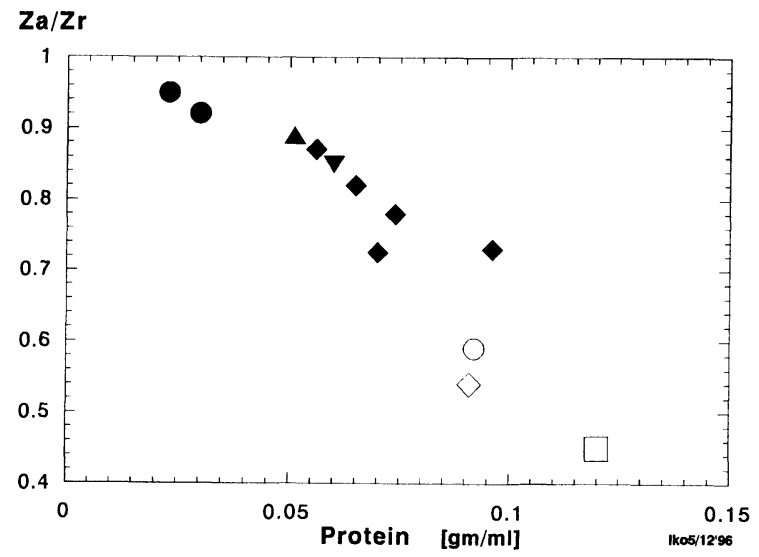

Fig. 4. Relative change of impedance. $\mathrm{Za}$, in activation. $\mathrm{Zr}$, in relaxation. Symbols are; =Actomyosin, $\boldsymbol{\Delta}$ =Myofilament,$\quad \boldsymbol{\nabla}=$ Minced muscle, $\boldsymbol{\nabla}=$ Muscle homogenate, $\diamond=$ Glycerinated MF. Values of muscle fiber $(\square)$ and skinned fiber $(\bigcirc)$ are plotted also.

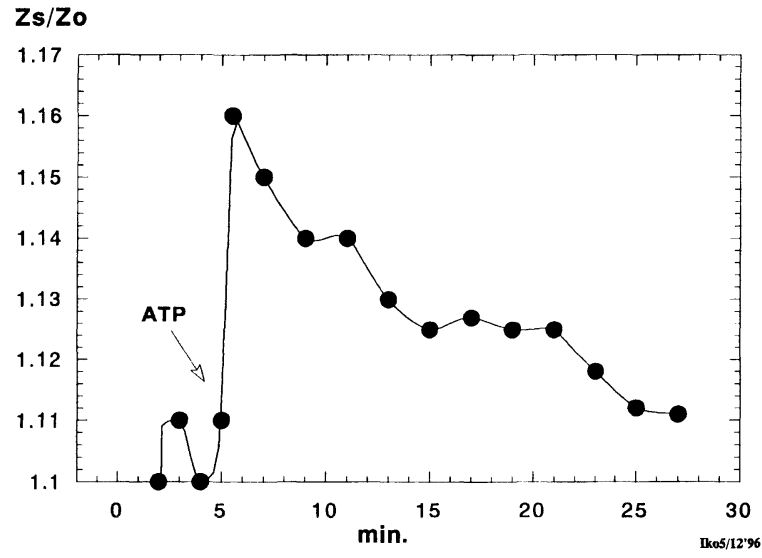

Fig. 5. ATP increases AM impedance. Zs, impedance of AM. Zo, impedance of stock solution.

conditions. The standard stock solution contains (in $\mathrm{mM}): \mathrm{KCl} 150, \mathrm{MgCl} 4$, EGTA 2, Tris-maleate $20(\mathrm{pH}$ 6.8). For the activation ATP $4-8 \mathrm{mM}$ and $\mathrm{CaCl}_{2} 2 \mathrm{mM}$ are applied. In each case the impedance of aqueous solution $\left(\mathrm{Z}_{0}\right)$ is measured for comparison.

$\mathrm{F}$-actin itself forms a solid hydrogel, and the ratio, $\left(\mathrm{Zs} / \mathrm{Z}_{0}\right)$, increases at the rate of $+0.025 / \mathrm{mgactin} / \mathrm{ml}$. Myosin sol is less viscous and its ratio is $+0.008 /$ mgmyosin/ml. Under the conditions without both ATP and $\mathrm{CaCl}_{2}$, the mixture of actin and myosin, (AM, weight ratio of $A: M, 1: 3)$, increases its impedance at a rate of $+0.008 / \mathrm{mgAM} / \mathrm{ml}$, almost the same as single myosin. Once ATP is applied to AM mixture, Zs increases within $30 \mathrm{sec},(69 \rightarrow 73 \Omega \mathrm{cm})$ despite the fact that $\mathrm{Z}_{0}$ decreases by $5 \%$. $\mathrm{Zs}$ is recovered gradually to an initial level with the consumption of ATP. (Fig. 5).

Glycerinated MF (rabbit psoas, $91 \mathrm{mg}$ protein $/ \mathrm{ml}$ ) suspended in the stock solution $\left(\mathrm{Z}_{0}, 64 \Omega \mathrm{cm}\right)$ shows $\mathrm{Zs}$ of $160 \Omega \mathrm{cm}$. After the application of ATP $(4 \mathrm{mM})$, Zs increases up to $177 \Omega \mathrm{cm},\left(\mathrm{Z}_{0}, 62.8 \Omega \mathrm{cm}\right)$. Then $\mathrm{Ca}^{2+}(2 \mathrm{mM})$ is added $\left(\mathrm{Z}_{0}, 61.4 \Omega \mathrm{cm}\right) .5$ minutes later Zs decreases down to $96 \Omega \mathrm{cm}$.

Change of the water structure in muscle. Dynamic ${ }^{1} \mathrm{H}-\mathrm{NMR}$ study showed that the water structure in muscle is more stable in relaxation than in tetanus (electrical stimulus, $100 \mathrm{~Hz}$, a few seconds). ${ }^{3)}$ At a magnetic field of $500 \mathrm{MHz}$ (FT-NMR) the bandshape of ${ }^{1} \mathrm{H}-\mathrm{NMR}$ for a frog skeletal muscle packed in a TEFLON tube without external solution, suggests two water groups (Wf, Ws) in muscle fiber.

Three successive signals obtained from the same sample are examined. Values of transverse relaxation time and population for Wf are $7.8 \mathrm{msec}$ and 0.70 respectively, before the stimulation. During tetanus $212 \mathrm{msec}$ and 0.865 , then $9.1 \mathrm{msec}$ and 0.74 after the stimulation. The value of transverse relaxation time for $\mathrm{Ws}$ is $2.3 \mathrm{msec}$ throughout the process. It seems therefore that water structure is stable in relaxation, and in contraction it becomes as if the water structure had collapsed.

Discussion. All the results presented in this article indicate that the water in the myoplasm is firmly retained by structural proteins, i.e., myosin, actin, and other related proteins, and does not move like ordinary water. If the muscle is activated, a considerable part of water can get rid of the constraint by structural proteins and becomes more movable.

The change certainly corresponds to the fall of electrical resistance during contraction discovered in $1965 .^{1,2)}$ Since then no report has been made concerning this and related problems.

The electrical impedance of actomyosin suspension increased on addition of ATP. This apparently seems to contradict to the above observation. However, the protein concentration in the suspension is low compared with that in living fibers. Since the interaction of actin and myosin is not only the function of their concentrations but also of ionic strength. If the actomyosin concentration is low, appropriate ionic strength becomes lower than so-called physiological concentration. The ionic strength used in this experiment was too high. This means that the increase in impedance of actomyosin suspension on addition of ATP was due to the solubilization of actomyosin as a result of its dissociation into actin and myosin. In other 
words the suspension becomes true solution of fairly high viscosity.

This is very suggestive of the situation in the myoplasm. In resting state, actin and myosin are almost in solubilized state and, therefore, the viscosity in the myoplasm is extremely high. On activation, actin and myosin interact with each other and forms a kind of solid gel. Now the water has become relatively free from actomyosin and can behave like ordinary water.

In the above, explanation was made as if the interaction of actin and myosin would be primary and the fall in electrical impedance might be secondary. However, water structure surrounding contractile proteins, especially that of actin, is very complex. Therefore, it is possible that the change in the water structure would take place concomitantly with the start of actin-myosin interaction. It may then not be so absurd to assume that the change in the water structure would be the primary event of contraction.

To verify which assumption would be reasonable, it is necessary to measure the change of impedance and other parameters at a very early stage of contraction, perhaps the change in millisecond after the onset of primary process. This is an extremely difficult experiment, but it must be performed to reveal the secret of muscle contraction.

The interaction of proteins and water is an important subject in biological science, particularly in the case where the proteins are involved in mechanical work. The phenomena presented in this article will bring forward an interesting problem in this direction.

Acknowledgments. Since 1965, the water has been my puzzling subject. First of all I would like to thank Prof. Setsuro Ebashi, M. J. A., and Dr. Fumiko Ebashi for their everlasting encouragement. I remember the stimulating discussions with Prof. John W. S. Pringle and Prof. Dr. Albert Szent-Györgyi.

\section{References}

1) Ogata, M. (1965) Abst. XXIII, I.C.P.S. $346 p$.

2) Ogata, M. (1968) Abst. XXIV, I.C.P.S. 325p

3) Ogata, M. (1992) J. Muscle Res. Cell Motil. 13, 479. 